\title{
Investigation in designing and developing the GNSS positioning instrumentation used in measurement and mapping by CORS/RTK technology
}

\author{
Khai Cong Pham ${ }^{1}{ }^{*}$, Hai Van Nguyen ${ }^{2}$, Nghia Viet Nguyen ${ }^{1}$ \\ ${ }^{1}$ Hanoi University of Mining and Geology, Hanoi, Vietnam \\ ${ }^{2}$ Thuyloi University, Hanoi, Vietnam
}

ARTICLE INFO ABSTRACT

Article history:

Received 05 ${ }^{\text {th }}$ Sept. 2021

Revised 23rd Dec. 2021

Accepted 28 ${ }^{\text {th }}$ Jan. 2022

Keywords:

GNSS receiver,

KX20 - R,

RTK KX Rover,

ZED - F9P.
Currently, in addition to GNSS receivers manufactured by foreign companies and imported into Vietnam, there are some domestically produced products with lower prices on the market. This paper presents an investigation on the design and development of a low-cost and highprecision GNSS device, used for CORS/RTK measurement in establishing topographic and cadastral maps. The developed GNSS device consists of a GNSS satellite antenna, a receiver, and an electronic field-book (using a smartphone device) installed with a self-developed software. The GNSS receiver is developed based on the technology and equipment of Drotek company (France). The components of the GNSS receiver have been selected and designed with functionality for both dynamic and static measurement modes. The GNSS receiver and electronic field-book are connected wirelessly via the Bluetooth module, allowing convenient operation with the receiver. Experiments were conducted to measure the parallel points of an established geodetic network using GPS technology. The possitioning data was measured by two receivers, South's S82 (China) and self-developed KX20-R, using RTK method with a single CORS station installed at the University of Mining and Geology. The results showed that the developed GNSS positioning device provides an accuracy of centimeters and completely meet the requirements of large-scale topographic and cadastral surveying and mapping.

Copyright (C) 2022 Hanoi University of Mining and Geology. All rights reserved.

${ }^{*}$ Corresponding author

E - mail: phamcongkhai@humg.edu.vn

DOI: 10.46326/JMES. 2022.63 (1).06 


\title{
Tạp chí Khoa học Kỹ thuật Mỏ - Địa chất
}

Trang điện tử: http://tapchi.humg.edu.vn

\section{Nghiên cứu thiết kế phát triển máy định vị vệ tinh GNSS trong đo đạc thành lập bản đồ theo kỹ thuật CORS/RTK}

\author{
Phạm Công Khải ${ }^{1}{ }^{*}$, Nguyễn Văn Hải ${ }^{2}$, Nguyễn Viết Nghĩa ${ }^{1}$ \\ 1 Trường Đại học Mỏ - Địa chất, Hà Nội, Việt Nam \\ ${ }^{2}$ Truờng Đại học Thủy Lợi, Hà Nội, Việt Nam
}

\begin{abstract}
THÔNG TIN BÀI BÁO
TÓM TẮT

Quá trình:

Nhận bài 05/9/2021

Sửa xong 23/12/2021

Chấp nhận đăng 28/01/2022

Tù̀ khóa:

Bộ thu GNSS,

KX20 - R,

RTK KX Rover,

ZED - F9P.

Hiện nay, ngoài các máy thu GNSS được sản xuất bởi các công ty nước ngoài và nhập khẩu vào Việt Nam, trên thị trường đã có một số sản phẩm được sản xuất ở trong nước với giá thành thấp hơn. Bài báo này trình bày nghiên cúu phát triển máy định vị GNSS giá thành thấp, có độ chính xác cao, sử dụng để đo đạc theo kỹ thuật CORS/RTK trong thành lập bản đồ địa hình và địa chính. Máy đinh vi GNSS đước phát triển gồm: ăng ten thu tín hiêu vê tinh GNSS, bô thu và sổ đo điện tư (sử dung smartphone) được cài đặt phần mềm tư thiết kế phát triển. Bồ thu GNSS được phát triển dựa trên công nghê và thiết bị của hãng Drotek (Cộng hòa Pháp). Các thành phần của bộ thu GNSS được lựa chọn và thiết kế với chức năng cho cả chế độ đo động và đo tĩnh. Máy thu GNSS và sổ đo điện tư được kết nối không dây thông qua module bluetooth cho phép thực hiện các thao tác thuận tiện với máy thu. Thực nghiệm đã được tiến hành đo vào các điểm song trùng của mạng lưới trắc địa đã được thành lập bằng công nghệ GPS. Sư dụng hai máy thu GNSS là S82 của hãng South (Trung Quốc) và máy KX20-R tự phát triển, đo theo phương thức RTK kết nối với trạm CORS đon lắp đặt ở Trường Đai hoc Mỏ - Đia chất. Kết quả cho thấy máy định vị GNSS được phát triển cho độ chính xác đến centimet, hoàn toàn đáp úng được cho cồng tác đo đạc thành lập bản đồ địa hình, địa chính tỷ lệ lớn.
\end{abstract}

(C) 2022 Trường Đại học Mỏ - Địa chất. Tất cả các quyền được bảo đảm.

\section{Mở đầu}

Ngày nay, các thiết bị đo đạc trắc địa ngày càng hoàn thiện nhờ áp dụng các thành tựu khoa học kỹ thuật. Sự phát triển của công nghệ hệ thống vệ tinh dẫn đường toàn cầu (GNSS), công tác định

*Tác giả liên hệ

E - mail: phamcongkhai@humg.edu.vn

DOI: 10.46326/JMES. 2022.63 (1).06 vị trên mặt đất sẽ được thực hiện bằng việc thiết lập mạng lưới các trạm tham chiếu hoạt động liên tục (CORS). Với mạng lưới trạm CORS, công tác đo đạc thành lập bản đồ hiện nay chủ yếu thực hiện theo phương thức đo động xử lý tức thời (RTK). Việc ứng dụng phương thức đo RTK sử dụng trạm CORS vào lĩnh vực trắc địa đã đem lại hiệu quả rất lớn trong việc thành lập bản đồ, rút ngắn thời gian đo đạc, giảm khối lượng công việc và cho độ chính xác cao (Pham \& Nguyen, 2019). 

Hiện nay, ở Việt Nam mạng lưới trạm định vị vệ tinh quốc gia (VNGEONET) đã được triển khai xây dựng làm cơ sở hạ tầng không gian quốc gia, người sử dụng sẽ được cung cấp nhiều ứng dụng, nhất là trong lĩnh vực đo đạc thành lập bản đồ. Để khai thác có hiệu quả mạng lưới trạm định vị vệ tinh quốc gia, người sử dụng chỉ cần một máy định vị GNSS là có thể thực hiện được công tác đo đạc thu thập số liệu thực địa, không phụ thuộc vào thời tiết và thời gian đo. Các máy định vị GNSS có trên thị trường ở Việt Nam hiện nay thường do các hãng sản xuất thiết bị đo đạc trên thế giới chế tạo như: Trimble (Mỹ), Leica (Thụy Sĩ), Topcon (Nhật Bản), South (Trung Quốc),... Tuy nhiên, các máy định vị này thường có giá bán khá cao, điều đó làm cho người sử dụng khó tiếp cận, làm giảm đi tính ứng dụng của công nghệ này.

Gần đây, nhiều hãng sản xuất các máy thu GNSS đã chuyển sang sản xuất các bảng mạch ở dạng sản xuất thiết bị gốc (Original Equipment Manufacturing - $\mathrm{OEM}$ ) để cung cấp cho người sử dụng tự phát triển thiết bị định vị, phục vụ cho những mục đích khác nhau. Trên thế giới, đã có nhiều nghiên cứu tập trung vào phát triển những thiết bị và giải pháp đo có hiệu quả cao, giá thành thấp. Chẳng hạn như: phát triển và đánh giá trạm tham chiếu ảo để định vị RTK đã được nghiên cứu bởi Hu và nnk. (2003) nhằm khai thác dữ liệu trạm CORS, nâng cao độ chính xác trạm động Rover; Hwang và nnk. (2012b) đã phát triển ứng dụng kỹ thuật định vị RTK - GPS và đã nghiên thành công cả về phần cứng và phần mềm cài đặt trong điện thoại thông minh; Trajkovski và nnk. (2010) đã nghiên cứu phát triển thiết bị định vị bằng cảm biến có độ nhạy cao trong điều kiện đo không thuận lợi; nghiên cứu của Lee (2010) đã tích hợp định vị GPS với cảm biến INS nhằm nâng cao độ chính xác đo động với cạnh cơ sở dài; các phương pháp tối ưu tích hợp RTK - GPS với gia tốc kế đã được Hwang và nnk. (2012a) phát triển để xác định sự dịch chuyển của các công trình; nghiên cứu gần đây, Manurung và nnk. (2019) ở Indonesia đã nghiên cứu phát triển bộ thu GNSS cho máy thu di động (Rover), đo được theo kỹ thuật CORS - RTK và có giá thành hợp lý. Ở Việt
Nam, trong thời gian gần đây cũng đã có một số công trình nghiên cứu phát triển bộ thu GNSS sử dụng trong quan trắc liên tục chuyển dịch biến dạng công trình theo thời gian thực (Phạm \& Trần, 2018). Việc thiết kế phát triển thiết bị định vị GNSS ứng dụng trong trắc địa bản đồ cũng đã được một số tổ chức, công ty thực hiện như: Trung tâm NAVIS của Đại học Bách khoa Hà Nội đã thực hiện đề tài nghiên cứu chế tạo hệ thống cung cấp dịch vụ định vị GPS độ chính xác centimet trong thời gian thực cho các lĩnh vực đòi hỏi độ chính xác định vị cao (Tạ, 2016); Trường Đại học Bách khoa Thành phố Hồ Chí Minh kết hợp với Công ty Đại Nam đã nghiên cứu phát triển một loại máy định vị P2 Elite GNSS hai tần số có độ chính xác cao. Công ty Cổ phần công nghệ hạ tầng cơ cở Aitogy đã phát triển một máy định vị Ainav - RTK - R được sử dụng để đo RTK theo công nghệ trạm CORS độ chính xác đến centimet. Tuy nhiên, máy định vị Ainav - RTK - R vẫn thiết kế chế tạo rời giữa ăng ten và bộ thu GNSS, các thành phần của máy còn tách rời nhau. Trong bài báo này, trình bày nghiên cứu phát triển máy định vị GNSS với mục tiêu thiết kế, phát triển được máy định vị vệ tinh GNSS mang nhãn hiệu Việt Nam có giá thành thấp, dễ sử dụng, đo được theo kỹ thuật CORS/RTK, đảm bảo yêu cầu độ chính xác, đáp ứng nhu cầu của người sử dụng ở Việt Nam trong công tác đo đạc thành lập bản đồ địa hình, địa chính.

\section{Thiết kế phát triển máy định vị GNSS}

\subsection{Các thành phần của máy định vị vệ tỉnh GNSS}

Trên thị trường hiện nay có nhiều máy định vị vệ tinh, mỗi loại máy có những đặc điểm và chức năng khác nhau để phù hợp với yêu cầu của người sử dụng. Theo cấu tạo, máy được chia làm hai loại là máy một tần số và hai tần số. Máy thu một tần số chỉ thu được một tín hiệu trên một tần số duy nhất L1. Máy thu hai tần số có thể thu đầy đủ tín hiệu trên hai tần số $L 1$ và $L 2$, điều này giúp cho máy hai tần số có phạm vi hoạt động rộng và độ chính xác cao hơn máy một tần số. Các máy định 

vị vệ tinh hai tần số gồm có các thành phần chính là ăng ten thu tín hiệu vệ tinh, bộ thu số liệu GNSS, sổ đo điện tử và các phụ kiện kèm theo. Ở Hình 1 là một máy định vị GNSS của hãng Trimble (Mỹ). Hiện nay, các hãng chế tạo đều sản xuất máy định vị hai tần số với chức năng đo tĩnh và đo động xử lý tức thời RTK.

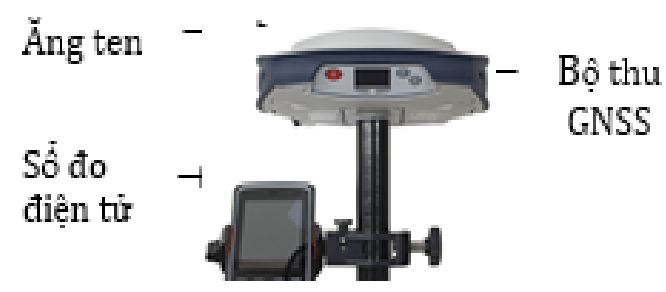

Hình 1. Máy định vị vệtinh GNSS của hãng Trimble (Mỹ).

\subsection{Thiết kế hệ thống phần cúng cho máy định vị GNSS}

Nền tảng công nghệ để thiết kế phát triển máy định vị GNSS là bảng mạch thu tín hiệu vệ tinh. Trên thế giới hiện nay, có nhiều hãng sản xuất bảng mạch để phát triển thiết bị định vị vệ tinh như: Trimble (Mỹ), Hemisphere (Mỹ), Drotek (Pháp), Tersus (Đài loan), NovAtel (Canada),... Việc lựa công nghệ và thiết bị để thiết kế phát triển bộ thu GNSS cho máy định vị RTK phụ thuộc vào yêu cầu công tác đo đạc, tính năng, tác dụng, độ chính xác và giá thành sản phẩm. Trong nghiên cứu này công nghệ và thiết bị được sử dụng để phát triển máy định vị GNSS là của hãng Drotek (Cộng hòa Pháp). Drotek là hãng sản xuất các thiết bị điện tử, các module ứng dụng trong lĩnh vực định vị vệ tinh. Các module chính được sử dụng để thiết kế phát triển máy định vị GNSS trong nghiên được thể hiện như ở Hình 2 và Bảng 1.
Bảng 1. Các module để phát triển máy định vị vệ tinh GNSS.

\begin{tabular}{|c|l|}
\hline TT & \multicolumn{1}{|c|}{ Tên thiết bị } \\
\hline 1 & Module định vị GNSS ZED - F9P \\
\hline 2 & Module xử lý dữ liệu Arduino UNO R3 \\
\hline 3 & Module thu nhận tín hiệu Max232 \\
\hline 4 & Module kết nối không dây Bluetooth \\
\hline 5 & Ăng ten GNSS \\
\hline
\end{tabular}

\subsubsection{Module định vị GNSS ZED - F9P}

Module định vị GNSS được sử dụng để thiết kế máy định vị có mã sản phẩm là DP0601 RTK ZED - F9P do hãng Drotek chế tạo (Hình 2a). Đây là module thu tín hiệu vệ tinh GNSS của các hệ thống GPS, COMPASS, LALILEO, COMPASS ở các tần số L1/L2. Module GNSS ZED - F9P có kích thước nhỏ gọn, tiêu thụ điện năng nhỏ, hỗ trợ nhận số cải chính theo định dạng RTCM nên phù hợp để phát triển máy thu GNSS sử dụng trong đo động xử lý tức thời (RTK) theo công nghệ CORS. Theo công bố của nhà sản xuất, module định vị GNSS ZED - F9P có sai số về mặt bằng là $10 \mathrm{~mm} \pm$ 1 ppm và độ cao là $15 \mathrm{~mm} \pm 1 \mathrm{ppm}$ (https://store - drotek.com/891 - rtk - zed - f9p - gnss.html).

Để sử dụng được module này cần phải cài đặt cấu hình bằng phần mềm "u - center" của nhà cung cấp thiết bị và được cài đặt vào máy tính. Module định vị GNSS ZED - F9P kết nối với máy tính thông qua cáp nối USB Micro - B (Hình 3).

\subsubsection{Module xư lý dũ liệu Arduino UNO R3}

Đây là module điều khiển trung tâm có nhiệm vụ điều khiển các module khác hoạt động, mọi mã code được nạp trực tiếp lên vi xử lý Atmega 328.

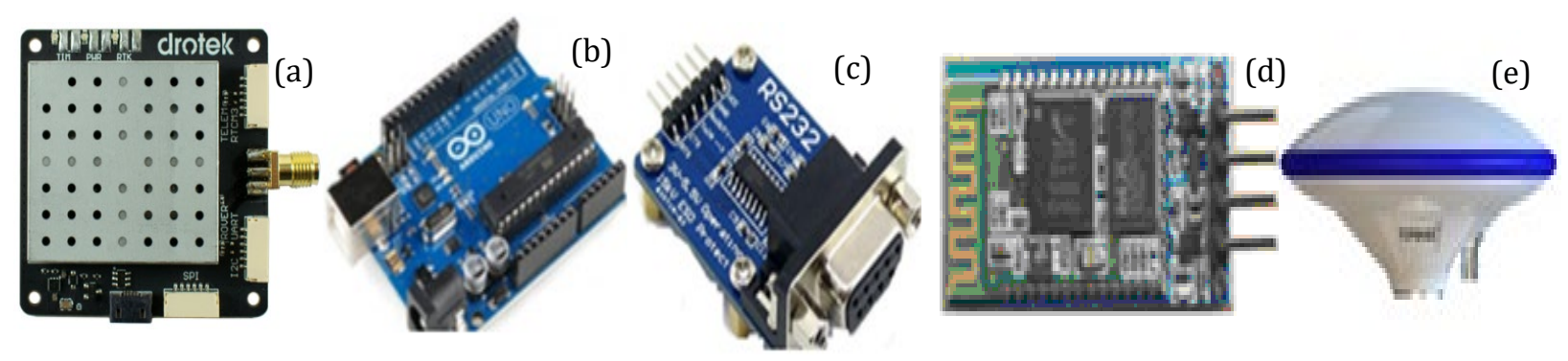

Hình 2. Một số module để phát triển máy định vị vệ tinh GNSS. 



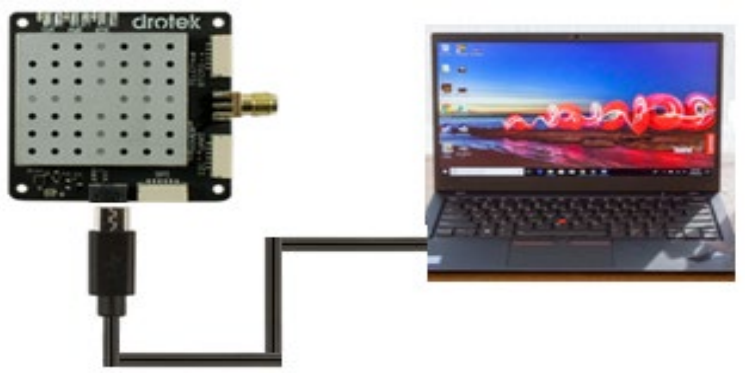

Hình 3. Kết nối để cài đặt cấu hình cho module GNSS ZED - F9P.

Trong các giao thức truyền dẫn tín hiệu, Atmega 328 có nhiệm vụ nhận dữ liệu tính toán và trả về các module kết nối các lệnh, các dữ liệu từ đây tạo thành các vòng kết nối liên tục và phụ thuộc vào nhau.

Module Arduino UNO R3 (Hình 2b) được thiết kế với 7 chân analog, 13 chân digital, 6/13 chân digital tích hợp. Bảng mạch chạy trong vùng điện áp trực tiếp từ $7 \mathrm{~V}$ đến $20 \mathrm{~V}$, chíp Atmega 328 là dòng chíp mới, họ $A V R$, hoạt động trên nền 8 bit, điện áp $5 \mathrm{~V}$, cường độ dòng $0,2 \mathrm{~mA}$, toàn bộ bảng mạch có mức tiêu thụ điện năng là $2,5 \mathrm{~W}$.

\subsubsection{Module thu nhận tín hiệu Max232}

Module thu nhận tín hiệu Max232 (Hình 2c) là thiết bị chuyển tín hiệu RS232 (Recommeded Standard 232) thành tín hiệu logic TTL (Transistor - Transistor Logic) để tạo sự giao tiếp giữa các thiết bị dùng chuẩn RS232 và thiết bị dùng chuẩn TTL. Đặc điểm của module Max232 là có độ chính xác cao, độ tin cậy về bảo toàn dữ liệu, tốc độ xử lý cao, dòng điện tiêu thụ và độ trễ tín hiệu nhỏ.

\subsubsection{Module bluetooth}

Module bluetooth có chức năng kết nối và giao tiếp giữa smartphone (sổ đo điện tử) với máy thu để truyền số liệu và cài đặt các thông số cho máy thu GNSS (Hình 2d).

\subsection{5. Ăng ten GNSS}

Ăng ten được sử dụng để thiết kế máy định vị GNSS có thương hiệu TOPGNSS, mã sản phẩm là GN - GGB0710 (Hình 2e), thu được tín hiệu vệ tinh GPS ở tần số L1, L2; Glonass G1, G2; Galileo L1/E5b/E5a; Beidou B1, B2, B3. Nguồn điện sử dụng $3.3 \div 18 \mathrm{~V}$. Ăng ten này có đặc điểm là độ chính xác cao khi đo RTK, giảm thiểu đáng kể sai số đa đường dẫn, có khả năng chống thấm nước và chống xốc.

\subsection{Thiết kế vỏ cho máy định vị GNSS}

Vỏ máy thu GNSS được thiết kế bằng phần mềm chuyên dụng sau đó được tạo hình bằng công nghệ in 3D như ở (Hình 4).

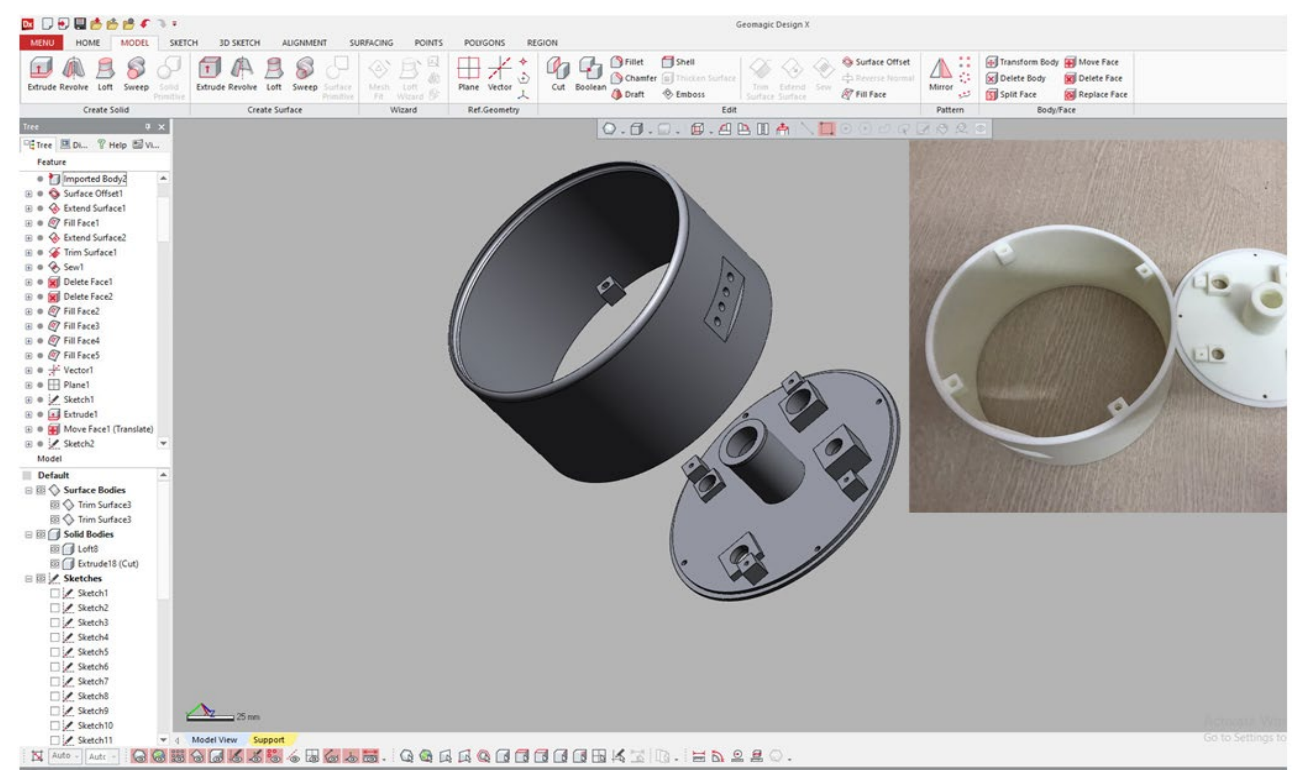

Hình 4. Vỏ máy GSNN được thiết kế và in 3D. 

Các module phần cứng được lắp ráp vào trong vỏ máy và hoàn thiện tạo thành máy định vị GNSS hoàn chỉnh với model KX20 - R như Hình 5.

\subsection{Thiết kế xây dụng phần mềm điều khiển}

Phần mềm điều khiển hoạt động của máy định vị GNSS được viết bằng ngôn ngữ C\# trong môi trường Android của điện thoại thông minh. Phần mềm có các chức năng: quản lý công việc đo, cài đặt các tham số tính chuyển tọa độ, cài đặt các thông số của trạm CORS, quản lý tệp số liệu. Phần mềm có tên là RTK KX Rover (Hình 6).

\section{3. Đánh giá độ chính xác máy định vị KX20-R}

Phương pháp để đánh giá độ chính xác của máy định vị vệ tinh GNSS đã được phát triển là sử dụng hai máy định vị đo vào cùng một điểm của mạng lưới khống chế đã xác định được tọa độ chính xác.

Mạng lưới khống chế (GPS - 1) dùng để đánh

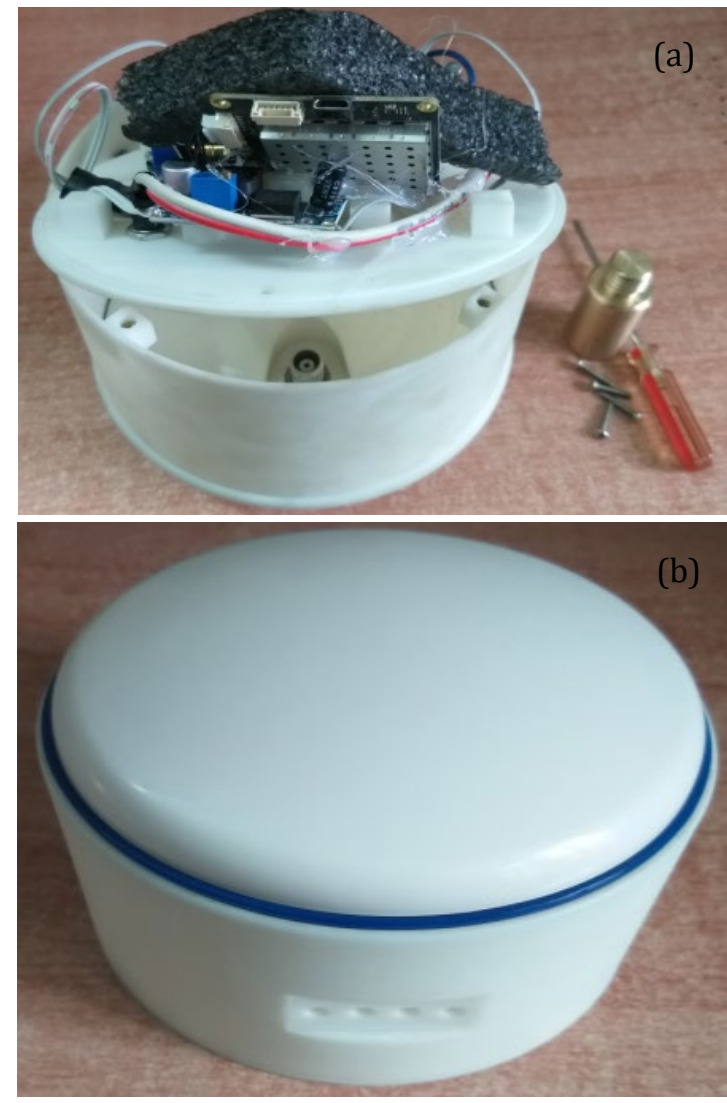

Hình 5. Máy định vị KX20-R được phát triển. (a)Đang hoàn thiện; (b)Đã hoàn thiện. giá độ chính xác của máy định vị GNSS KX20 - R được thành lập bằng công nghệ GPS (Hình 7) và được bình sai bằng phần mềm TBC 3.5. Kết quả bình sai tính toán, xác định được tọa độ của các điểm trong mạng lưới cũng như sai số của nó được thể hiện ở Bảng 2.

Sử dụng hai máy đo GNSS KX20 - R và máy S82 của hãng South. Máy S82 là dòng máy định vị GNSS hai tần số thu được 220 kênh của các hệ thống vệ tinh GPS, Glonass, Compass. Độ chính xác khi đo RTK về mặt bằng là $10 \mathrm{~mm} \pm 1 \mathrm{ppm}$ và về độ cao là $15 \mathrm{~mm} \pm 1 \mathrm{ppm}$. Phương thức truyền thông qua USB, Bluetooth, các cổng nối tiếp RS 232. Như vậy, máy $S 82$ khá tương đồng với máy KX20 - R, do đó nó được lựa chọn để đo kiểm chứng, hơn nữa máy $S 82$ có giá thành thấp, dễ tìm trên thị trường ở Việt Nam. Khi tiến hành thực nghiệm, sử dụng hai máy S82 và KX20 - R lần lượt đặt vào các điểm của mạng lưới kiểm định (Hình 7) và đo theo kỹ thuật CORS/RTK, sử dụng trạm

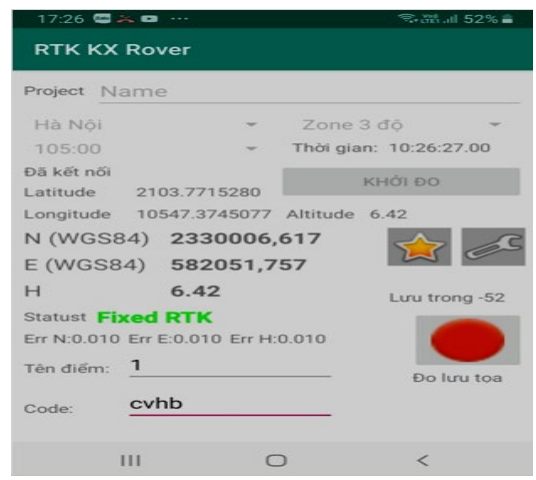

Hình 6. Giao diện phần mềm RTK KX Rover cài đặt trong điện thoại thông minh.

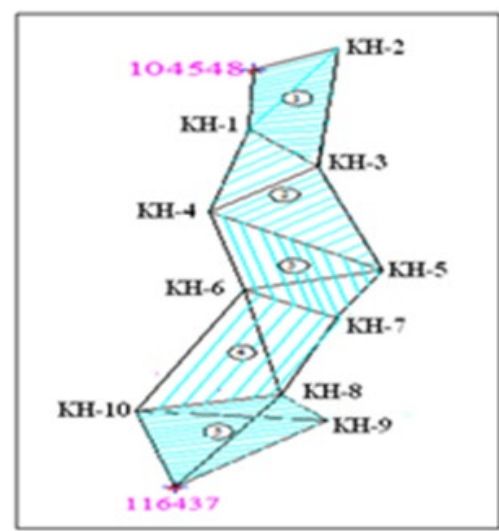

Hình 7. Mạng lưới GPS - 1 để đánh giá độ chính xác của máy định vị vệ tinh KX20 - R. 

CORS đơn của Leica lắp đặt tại Trường Đại học Mỏ - Địa chất. Trạm CORS này cùng loại với các trạm CORS đã được xây dựng trên lãnh thổ Việt Nam và được Cục Đo đạc Bản đồ và thông tin Địa lý Việt Nam quản lý, vận hành. Hình ảnh đo đạc thực nghiệm để đánh giá độ chính xác của máy định vị GNSS KX20 - R được thể hiện như ở Hình 8.

Từ tọa độ của các điểm trong mạng lưới xác định được bằng hai máy đo là S82 và máy KX20 $\mathrm{R}$, dựa vào tọa độ đã biết của các điểm đó sẽ xác định được sai lệch về tọa độ mặt bằng của hai loại máy được thể hiện như ở các Bảng 3, 4 và được biểu thị sai số về mặt bằng như ở Hình 9.

Biểu đồ Hình 9 nhận thấy, sai số về tọa độ mặt bằng khi đo bằng hai máy có giá trị xấp xỉ nhau và đều tăng dần khi khoảng cách từ trạm CORS đến máy đo tăng lên. Sai số lớn nhất tại điểm KH - 9 khi đo bằng máy $\mathrm{S} 82$ là $0,064 \mathrm{~m}$ và khi đo bằng máy KX20 - R là 0,068 m.

Bảng 2. Tọa độ của các điểm sau bình sai của mạng luới GPS-1. Hệ tọa độ: VN 2000;

Kinh tuvến truc: $105.00-Z 3$.

\begin{tabular}{|c|c|c|c|c|c|}
\hline Điểm & $\mathrm{X}(\mathrm{m})$ & $\mathrm{Y}(\mathrm{m})$ & $\mathrm{M}_{\mathrm{P}}(\mathrm{m})$ & $\mathrm{h}(\mathrm{m})$ & $\mathrm{M}_{\mathrm{h}}(\mathrm{m})$ \\
\hline 104548 & 2332590,893 & 581018,697 & --- & 7,977 & --- \\
\hline 116437 & 2321949,130 & 579987,381 & ---- & 6,731 & ---- \\
\hline KH-1 & 2331078,161 & 580952,520 & 0,003 & 7,242 & 0,008 \\
\hline KH-2 & 2333147,615 & 582109,681 & 0,004 & 15,608 & 0,007 \\
\hline KH-3 & 2330112,400 & 581855,510 & 0,003 & 6,567 & 0,009 \\
\hline KH-4 & 2328976,440 & 580430,385 & 0,004 & 5,955 & 0,010 \\
\hline KH-5 & 2327466,300 & 582693,099 & 0,004 & 6,144 & 0,013 \\
\hline KH-6 & 2326970,116 & 580887,986 & 0,004 & 6,744 & 0,014 \\
\hline KH-7 & 2326251,236 & 582094,451 & 0,004 & 6,615 & 0,014 \\
\hline KH-8 & 2324291,465 & 581377,192 & 0,004 & 6,002 & 0,010 \\
\hline KH-9 & 2323643,828 & 581962,164 & 0,007 & 6,089 & 0,018 \\
\hline KH-10 & 2323870,602 & 579504,400 & 0,004 & 6,654 & 0,008 \\
\hline
\end{tabular}
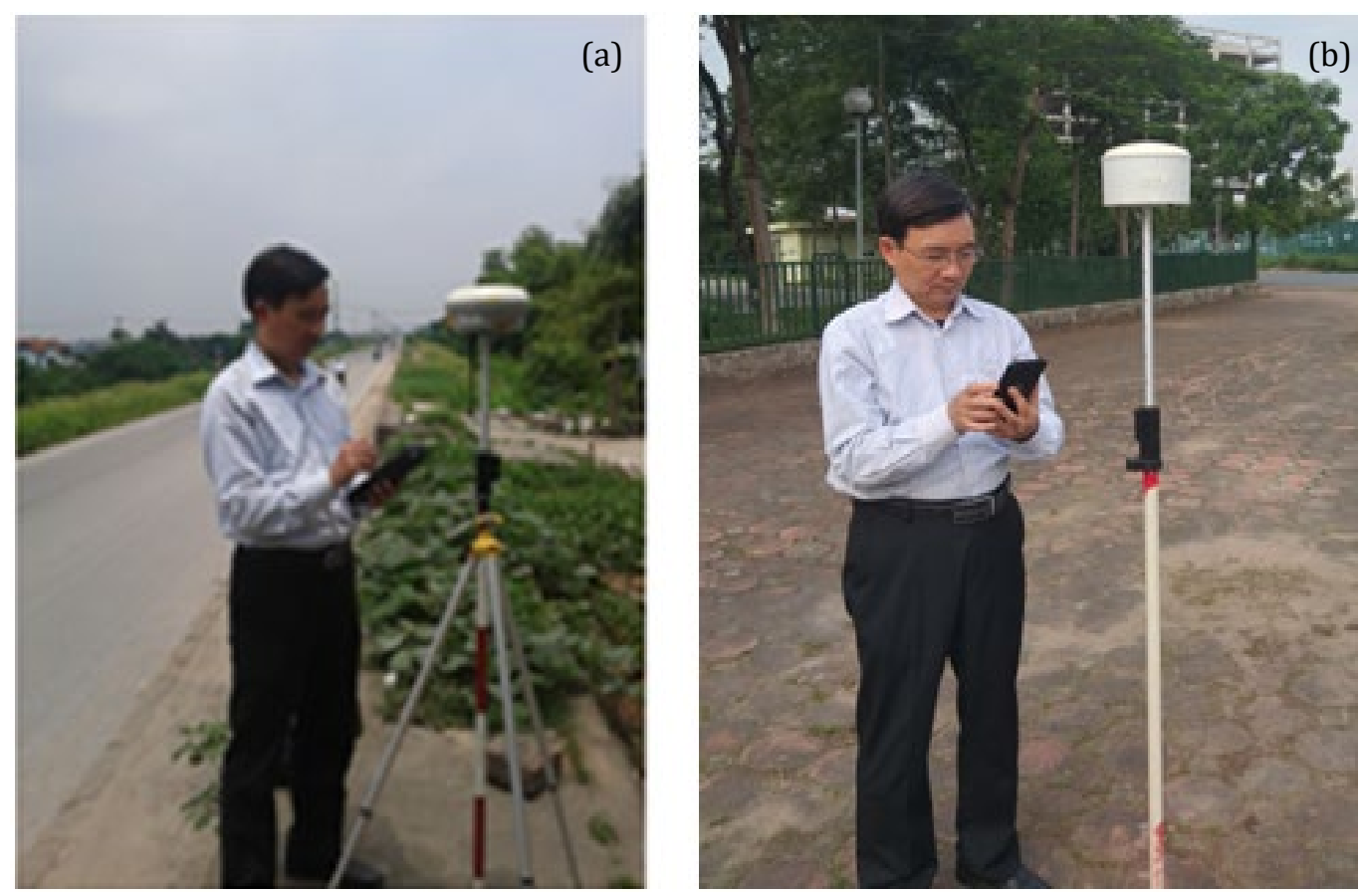

Hình 8. Đo thực nghiệm đánh giá độ chính xác của máy KX20-R. (a) Đo bằng máy S82; (b) Đo bằng máy KX20-R. 
Bảng 3. Sai lệch tọa độ mặt bằng khi đo bằng máy KX20 - R.

\begin{tabular}{|c|c|c|c|c|c|c|c|c|c|}
\hline \multirow[t]{2}{*}{ TT } & \multirow[t]{2}{*}{ Điểm } & \multicolumn{2}{|c|}{ Tọa độ gốc (m) } & \multicolumn{2}{|c|}{$\begin{array}{c}\text { Tọa độ đo bằng máy } \\
\text { KX20-R (m) }\end{array}$} & \multicolumn{2}{|c|}{ Sai lệch tọa độ (m) } & \multirow{2}{*}{$\begin{array}{c}\text { Sai số vị trí } \\
\text { mặt bằng } \\
\text { MP (m) }\end{array}$} & \multirow{2}{*}{$\begin{array}{l}\text { Khoảng cách từ } \\
\text { trạm CORS đến } \\
\text { điểm đo }(\mathrm{km})\end{array}$} \\
\hline & & $\mathrm{X}_{\text {gốc }}$ & $Y_{\text {gốc }}$ & $\mathrm{X}_{\mathrm{RTK}}$ & $\mathrm{Y}_{\mathrm{RTK}}$ & $\sigma \mathrm{X}$ & $\sigma \mathrm{Y}$ & & \\
\hline 1 & $\mathrm{KH}-1$ & 2331078,161 & 580952,520 & 2331078,153 & 580952,529 & $-0,008$ & 0,009 & 0,012 & 1,01 \\
\hline 2 & $\mathrm{KH}-4$ & 2328976,440 & 580430,385 & 2328976,428 & 580430,371 & $-0,012$ & $-0,014$ & 0,018 & 1,30 \\
\hline 3 & $\mathrm{KH}-3$ & 2330112,400 & 581855,510 & 2330112,386 & 581855,494 & $-0,014$ & $-0,016$ & 21 & 1,51 \\
\hline 4 & KH-6 & 2326970,116 & 580887,986 & 2326970,135 & 580888,008 & 0,019 & 0,022 & 0,029 & 3,35 \\
\hline 5 & KH-2 & 2333147,615 & 582109,681 & 2333147,640 & 582109,708 & 0,025 & 0,027 & & 3,37 \\
\hline 6 & KH-5 & 2327466,300 & 582693,099 & 2327466,273 & 582693,068 & $-0,027$ & $-0,031$ & 0,041 & 3,66 \\
\hline 7 & KH-7 & 2326251,236 & 582094,451 & 2326251,208 & 582094,486 & $-0,028$ & 0,035 & 45 & 4,38 \\
\hline 8 & KH-8 & 2324291,465 & 581377,192 & 2324291,429 & 581377,231 & $-0,036$ & 0,039 & 0,053 & 6,07 \\
\hline 9 & $\mathrm{KH}-10$ & 2323870,602 & 579504,400 & 2323870,559 & 579504,354 & $-0,043$ & $-0,046$ & 0,063 & 6,46 \\
\hline 10 & KH-9 & 2323643,828 & 581962,164 & 2323643,781 & 581962,213 & $-0,047$ & 0,049 & 0,068 & 6,82 \\
\hline
\end{tabular}

Bảng 4. Sai lệch tọa độ mặt bằng khi đo bằng máy S82 của South.

\begin{tabular}{|c|c|c|c|c|c|c|c|c|c|}
\hline \multirow[t]{2}{*}{ No } & \multirow[t]{2}{*}{ Điểm } & \multicolumn{2}{|c|}{ Tọa độ gốc (m) } & \multicolumn{2}{|c|}{ Tọa độ đo bằng máy S82 (m) } & \multicolumn{2}{|c|}{$\begin{array}{l}\text { Sai lệch tọa độ } \\
\text { (m) }\end{array}$} & \multirow{2}{*}{$\begin{array}{c}\text { Sai số vị trí } \\
\text { mặt bằng } \\
\text { MP (m) }\end{array}$} & \multirow{2}{*}{$\begin{array}{l}\text { Khoảng cách tù̀ } \\
\text { trạm CORS đến } \\
\text { điểm đo }(\mathrm{km})\end{array}$} \\
\hline & & $\mathrm{X}_{\text {gốc }}$ & Ygốc & $\mathrm{X}_{\mathrm{RTK}}$ & $\mathrm{Y}_{\mathrm{RTK}}$ & $\sigma \mathrm{X}$ & $\sigma \mathrm{Y}$ & & \\
\hline 1 & KH-1 & 2331078,161 & 580952,520 & 2331078,157 & 580952,526 & $-0,004$ & 0,006 & 0,007 & 1,01 \\
\hline 2 & KH-4 & 2328976,440 & 580430,385 & 2328976,432 & 580430,376 & $-0,008$ & $-0,009$ & 0,012 & 1,30 \\
\hline 3 & KH-3 & 2330112,400 & 581855,510 & 2330112,410 & 581855,524 & 0,010 & 0,014 & 0,017 & 1,51 \\
\hline 4 & KH-6 & 2326970,116 & 580887,986 & 2326970,095 & 580888,008 & $-0,021$ & 0,022 & 0,030 & 3,35 \\
\hline 5 & $\mathrm{KH}-2$ & 2333147,615 & 582109,681 & 2333147,635 & 582109,702 & 0,020 & 0,021 & 0,029 & 3,37 \\
\hline 6 & KH-5 & 2327466,300 & 582693,099 & 2327466,278 & 582693,076 & $-0,022$ & $-0,023$ & 0,032 & 3,66 \\
\hline 7 & KH-7 & 2326251,236 & \begin{tabular}{|l|}
582094,451 \\
\end{tabular} & 2326251,207 & 582094,480 & $-0,029$ & 0,029 & 0,041 & 4,38 \\
\hline 8 & KH-8 & 2324291,465 & 581377,192 & 2324291,425 & 581377,235 & $-0,040$ & 0,043 & 0,059 & 6,07 \\
\hline 9 & $\mathrm{KH}-10$ & 2323870,602 & \begin{tabular}{|l|}
579504,400 \\
\end{tabular} & 2323870,560 & 579504,356 & $-0,042$ & $-0,044$ & 0,061 & 6,46 \\
\hline 10 & KH-9 & 2323643,828 & 581962,164 & 2323643,786 & 581962,212 & $-0,042$ & 0,048 & 0,064 & 6,82 \\
\hline
\end{tabular}

BIÉU ĐỎ SO SÁNH ĐỌ̄ CHÍNH XÁC TỌA ĐỌ̄ MẠ̄T BẢNGCỦA HAI MÁY ĐO S82 VÀ KX20-R

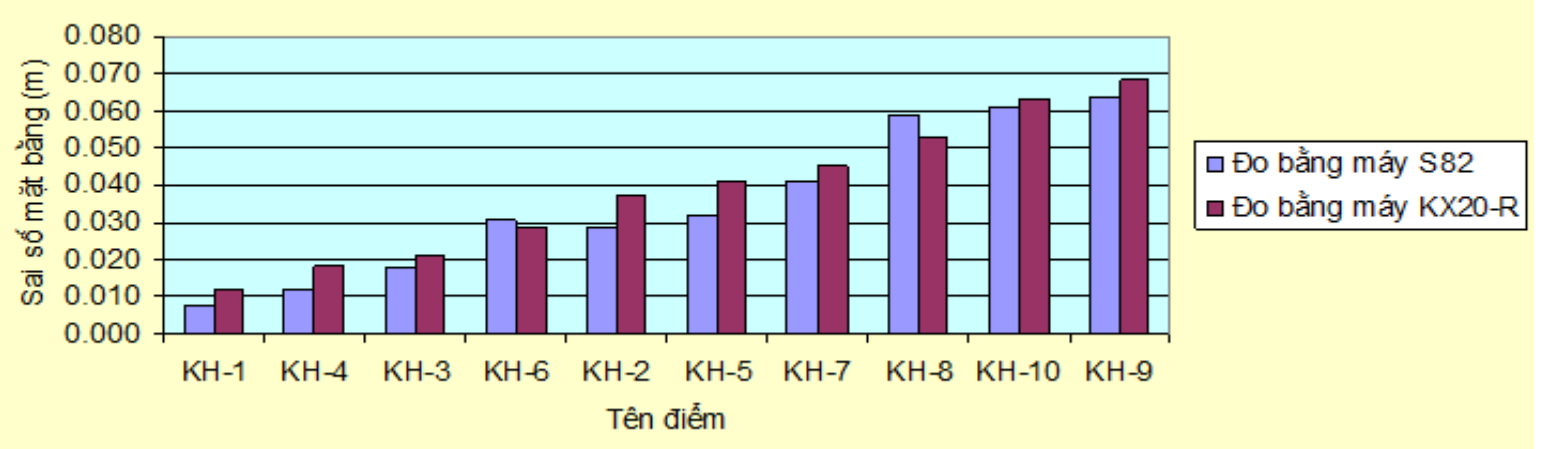

Hình 9. Biểu đồ so sánh độ chính xác tọa độ mặt bằng của hai máy đo S82 và KX20-R.

Như vậy có thể nhận thấy rằng giữa sai số vị trí điểm đo bằng kỹ thuật CORS/RTK và khoảng cách từ trạm CORS đến máy đo GNSS có mối quan hệ với nhau (Pham \& Nguyen, 2019).

Dựa vào số liệu ở Bảng 3 khi đo bằng máy KX20-R, xây dựng được phương trình hồi quy biểu thị mối quan hệ giữa sai số mặt bằng và khoảng cách được thể hiện như (Hình 10).
Phương trình hồi quy biểu diễn mối quan hệ giữa sai số vị trí mặt bằng (biến phụ thuộc) và khoảng cách từ trạm CORS đến máy định vị GNSS (biến độc lập), có dạng như phương trình (1):

$$
\begin{gathered}
M_{P}=0.009 D+0.0024 \\
R^{2}=0.981
\end{gathered}
$$

Trong đó: $M_{P}$ - sai số vị trí điểm (m); 
$D$ - khoảng cách từ trạm CORS đến trạm động Rover (km); R - Hệ số tương quan.

Dựa vào phương trình hồi quy (1) sẽ xác định được khoảng cách lớn nhất từ trạm CORS đến máy định vị khi áp dụng kỹ thuật đo CORS/RTK để đảm bảo độ chính xác khi thành lập bản đồ địa chính tỷ lệ lớn theo công thức (2).

$$
\mathrm{D}_{\mathrm{PMax}}=\frac{\left(\left[\mathrm{M}_{\mathrm{P}}\right]-0.0024\right)}{0.009}
$$

Trong đó: $\mathrm{D}_{\mathrm{Max}}$ - khoảng cách lớn nhất từ trạm CORS đến máy định vị GNSS; $\left[\mathrm{M}_{\mathrm{P}}\right]$ - sai số vị trí điểm chi tiết khi đo bằng kỹ thuật CORS/RTK.

Sai số trị trí điểm chi tiết cho phép lớn nhất cho phép (Thông tư 68/2015/TT-BTNMT) được tính theo công thức (3).

$$
\left[M_{P}\right]=0.1 \times M(\mathrm{~mm})
$$

Trong đó: $M$ - mẫu số tỷ lệ bản đồ địa chính cần thành lập.

Dựa vào các công thức (2) và (3), xác định được khoảng cách lớn nhất từ trạm CORS đến trạm động Rover khi đo bằng kỹ thuật CORS/RTK như ở Bảng 5.
Như vậy, với máy KX20 - R đã được thiết kế phát triển hoàn toàn đáp ứng cho việc đo vẽ bản đồ địa chính tỷ lệ lớn bằng kỹ thuật đo CORS/RTK.

\section{Kết luận}

Trong nghiên cứu này đã tập trung vào giải quyết vấn đề thiết kế phát triển máy định vị GNSS đo theo kỹ thuật CORS/RTK có độ chính xác đến centimet và giá thành thấp, đáp ứng được trong công tác đo đạc thành lập bản đồ tỷ lệ lớn.

Một bộ thu GNSS đã được thiết kế phát triển và chế tạo dựa trên nền tảng công nghệ, thiết bị của hãng Drotek và phần mềm RTK KX Rover được thiết kế xây dựng và cài đặt trên thiết bị di động thông minh với các chức năng quản lý công việc đo, cài đặt các tham số tính chuyển tọa độ và các thông số của trạm CORS, quản lý tệp số liệu đo tạo nên một máy định vị GNSS hoàn chỉnh.

Kết quả đo thử nghiệm bằng hai máy định vị S82 và máy khác KX20 - $\mathrm{R}$ cho thấy máy định vị KX20 - R được thiết kế phát triển đảm bảo yêu cầu độ chính xác, đáp ứng được cho công tác đo đạc thành lập bản đồ địa chính tỷ lệ lớn.

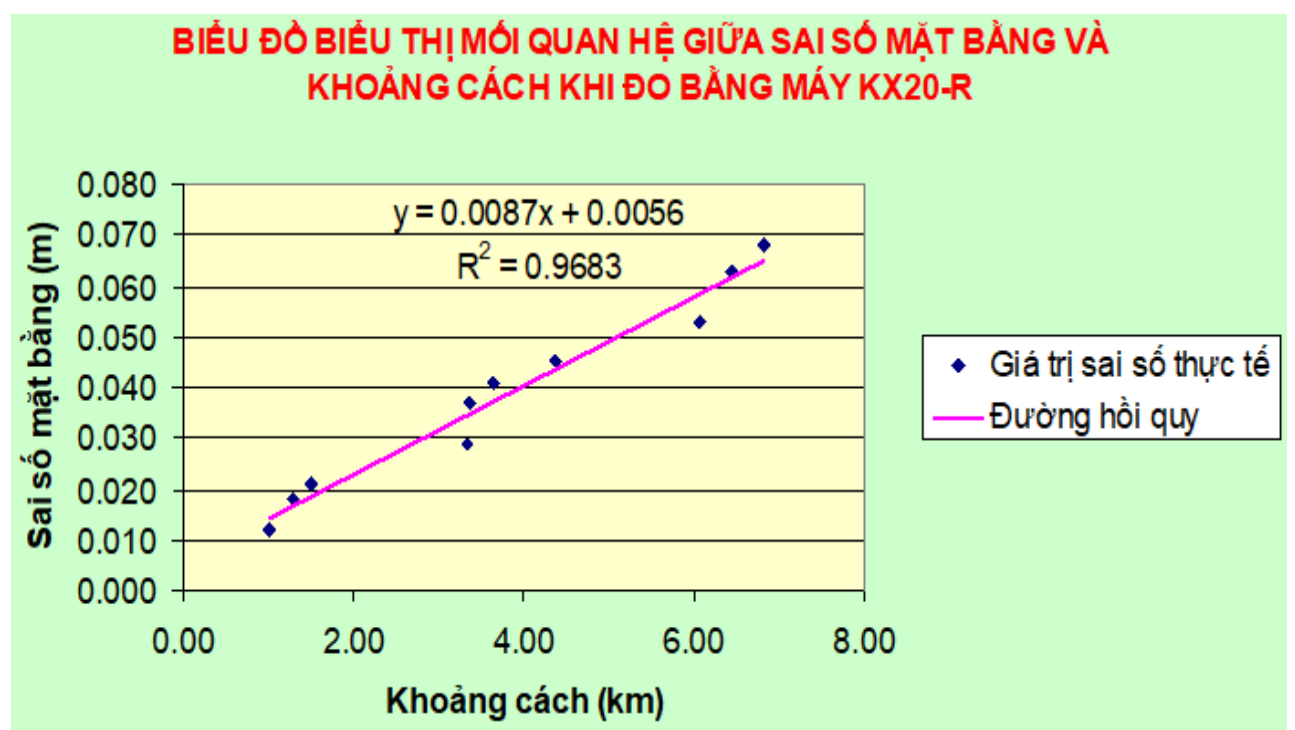

Hình 10. Mối quan hệ giữa sai số mặt bằng và khoảng cách.

Bảng 5. Khoảng cách lớn nhất tù trạm CORS đến trạm động Rover khi đo vẽ bản đồ địa chính bằng máy $K X 20$ - $R$

\begin{tabular}{|l|c|c|c|c|}
\hline \multicolumn{1}{|c|}{ Tỷ lệ bản đồ } & $1: 500$ & $1: 1000$ & $1: 2000$ & $1: 5000$ \\
\hline Sai số vị trí điểm cho phép (m) & 0,08 & 0,15 & 0,30 & 0,75 \\
\hline Khoảng cách đo lớn nhất (km) & 5,3 & 13,4 & 29,5 & 54,4 \\
\hline
\end{tabular}




\section{Các đóng góp của các tác giả}

Phạm Công Khải - lên ý tưởng, xây dựng đề cương, đọc bản thảo bài báo và viết bản thảo; Nguyễn Văn Hải - thu thập số liệu; Nguyễn Viết Nghĩa - triển khai thực nghiệm, xử lý số liệu, cho các ý kiến góp ý.

\section{Tài liệu tham khảo}

https://store - drotek.com/891 - 1023 - rtk - zed f9p - gnss.html\#/105 - case - without.

Hu, G. R., Khoo, H. S., Goh, P. C., \& Law, C. L. (2003). Development and assessment of GPS virtual reference stations for RTK positioning. Journal of Geodesy, 77(5), 292-302.

Hwang, J., Yun, H., Park, S. K., Lee, D., \& Hong, S. (2012a). Optimal methods of RTKGPS/accelerometer integration to monitor the displacement of structures. Sensors, 12(1), 1014-1034.

Hwang, J., Yun, H., Suh, Y., Cho, J., \& Lee, D. (2012b). Development of an RTK-GPS positioning application with an improved position error model for smartphones. Sensors, 12(10), 12988-13001.

Lee, H. (2010). An integration of GPS with INS sensors for precise long-baseline kinematic positioning. Sensors, 10(10), 9424-9438.
Manurung, P., Pramujo, H., \& Manurung, J. B. (2019). Development of GNSS receiver for mobile CORS with RTK correction services using cloud server. In E3S Web of Conferences (Vol. 94, p. 01010). EDP Sciences.

Pham, C. K., Nguyen, Q. L. (2019). Accuracy assessment of the single CORS technology for establishing the large scale cadastral map. International Journal of Scientific \& Engineering Research, 10(4). 113-123. ISSN 2229-5518.

Phạm, C. K., Trần, T. X. (2018). Nghiên cứu phát triển hệ thống quan trắc chuyển dịch biến dạng công trình theo thời gian thực. Tạp chí công nghiệp mỏ, 4, 33-38.

Tạ, H. T. (2016). Nghiên cúu chế tạo hệ thống cung cấp dịch vụ định vị GPS độ chính xác cm trong thời gia thực cho các lĩnh vực đòi hỏi độ chính xác cao. Báo cáo kết quả nghiên cứu đề tài, mã số VT/CN - 02/13-15

Trajkovski, K. K, Sterle, O., \& Stopar, B. (2010). Study positioning with high sensitivity GPS sensors under adverse conditions. Sensors, 10, 8332-8347.

Thông tư 68/2015/TT-BTNMT. (2015). Quy định kỹ thuật đo đạc trực tiếp địa hình phục vu thành lập bản đồ địa hình và cơ sở dũ liệu nền địa lý tỷ lệ 1:500, 1:1000, 1:2000, 1:5000. 